\title{
RESULTS FROM TERRASAR-X GEOMETRIC AND RADIOMETRIC CALIBRATION
}

\author{
B. Bräutigam, M. Schwerdt, M. Bachmann, B. Döring \\ German Aerospace Center (DLR), Microwaves and Radar Institute, Oberpfaffenhofen, D-82234 Wessling, Germany \\ Benjamin.Braeutigam@dlr.de
}

Keywords: TerraSAR-X, Calibration, Antenna Model.

\begin{abstract}
As TerraSAR-X, due for launch in June 2007, will be an operational scientific mission with commercial potential, product quality is of crucial importance. The success or failure of the mission essentially depends on the calibration of the TerraSAR-X system ensuring the product quality and the correct in-orbit operation of the entire SAR system.

This paper describes the calibration procedures for TerraSAR-X and the dedicated activities to be performed during the five months commissioning phase. Results from on-ground tests are discussed with respect to geometric and radiometric calibration of the TerraSAR-X system.
\end{abstract}

\section{Introduction}

The first German SAR satellite for commercial and scientific applications, TerraSAR-X, will launch in June 2007. TerraSAR-X is a flexible $\mathrm{X}$-Band SAR built by EADS Astrium and the German Aerospace Center (DLR) in a public-private-partnership [1]. The SAR instrument is designed to cover a wide range of swath positions and to operate in different operation modes by switching the instrument over a multitude of antenna beams. For the various antenna beams, an active phased array antenna electronically forms antenna radiation patterns. The array consists of 384 radiating sub-arrays for horizontal and vertical polarisation arranged in a matrix of 12 panels with 32 rows. Active transmit/receive (T/R) modules individually adjust the array elements in gain and phase for shaping and steering the antenna pattern in azimuth and elevation direction.

The essential task of calibrating TerraSAR-X is to estimate and correct systematic error contributions throughout the complete SAR system and to tie-down image information (magnitude and phase) to reference units in geophysical terms. The quality of this calibration process depends on the inherent stability of the radar system and the capability to determine and monitor the radiometric and geometric characteristics [2,3,4].

Due to the high degree of flexibility of TerraSAR-X (StripMap, Spotlight, ScanSAR, right/left looking, etc.) and a tight performance with an absolute radiometric accuracy better than $1 \mathrm{~dB}$, it becomes clear that a conventional calibration approach is not feasible, i.e. the real measurement of all antenna beams in all operation modes, as performed for the ASAR instrument of ENVISAT across the rainforest [5]. Hence, a new, more efficient, and affordable calibration concept has been developed $[3,4]$. The key element of this calibration concept is a novel antenna model approach $[4,6]$. The TerraSAR-X antenna model is utilised for generating all reference antenna patterns and for beam optimisation.

To account for the restricted time of calibration campaigns performed during commissioning phase of TerraSAR-X, the number of passes and places of test sites is optimised versus cost and time effort by calibrating only dedicated beams but with the same test site.

The paper describes the in-orbit calibration procedure and the different activities performed before and after launch of TerraSAR-X. It shows the calibration status until satellite launch and gives an overview of the planned activities during the five months commissioning phase.

\section{Scope of TerraSAR-X Calibration}

Applying the novel antenna model approach for SAR system calibration, the objective of in-orbit calibration can be subdivided into three major tasks:

- Geometric Calibration: to assign the SAR system to the geographic location on the earth surface.

- Relative Radiometric Calibration: for radiometric correction of SAR data within an illuminated scene.

- Absolute Radiometric Calibration: the measurement of the SAR system against standard targets with well known geophysical characteristics.

The calibration steps rely on the antenna model which has been validated on-ground. In addition to the tasks described above, the verification of this antenna model has to be executed in-orbit. This ensures the provision of the antenna patterns of all operation modes and the gain offset between adjoining beams.

\section{Strategy}

The main goal after launch of the TerraSAR-X satellite is to provide calibrated and verified SAR data products at the latest by the end of the commissioning phase. Thus, a strategy for an efficient but robust calibration approach has been developed. 
The successive baseline calibration processes are:

\section{Geometric Calibration \\ 2. Antenna Pointing Determination \\ 3. Antenna Model Verification \\ 4. Relative Radiometric Calibration \\ 5. Absolute Radiometric Calibration}

Furthermore, by applying the novel T/R Module Characterisation Method [7,8], the actual antenna settings will be derived in orbit. Individual and simultaneous monitoring of all $\mathrm{T} / \mathrm{R}$ modules is possible in-flight under the most realistic instrument conditions. This saves valuable time during commissioning and in case of antenna module failure or degradation.

In the following the process steps of the in-orbit calibration are described in detail.

\subsection{Geometric Calibration}

The purpose of the geometric calibration is the geometric assignment of the SAR system to the earth's surface. Two effects can influence the correct localisation of the product:

- systematic azimuth shifts resulting in wrong data take start time and

- internal electronic delay of the instrument dislocating products in range.

For both effects, SAR measurements over corner reflectors are analysed as passive targets have no additional electronic delay and consequently no additional source of errors.

\subsection{Antenna Pointing Determination}

An important task is the determination of beam pointing errors coming from mechanical and electrical antenna mispointing as well as from attitude control offsets. These errors are measured in elevation and in azimuth using appropriate patterns over rain forest and by ground receivers. But also a Doppler analysis is performed to evaluate a squint in flight direction.

\subsection{Antenna Model Verification}

The total size of the SAR antenna is $4.8 \mathrm{~m}$ in length and $0.8 \mathrm{~m}$ in height. Far-field pattern measurement of the complete antenna was not possible on-ground due to restricted space in the measurement chamber. Real pattern measurements can only be performed in orbit.

The characterisation of the antenna is based on a precise antenna pattern model, which had to be validated against precisely measured near-field patterns on ground before launch and will be verified in orbit by in-flight measurements over homogeneous targets (rain forest) and deployed ground receivers.

The antenna model provides a software tool that accurately determines all beam patterns based on detailed characterisation of the radar antenna and knowledge of the antenna control parameters.

It was developed before launch considering different input parameters like:

- geometry of the antenna,

- beam excitation coefficients of all $384 \mathrm{~T} / \mathrm{R}$ modules,

- a drift and failure matrix and

- embedded radiation patterns measured on ground from single sub-arrays of the antenna.

The strategy of on-ground model validation was based on the comparison between simulations and measurements.

For this purpose, the antenna patterns of the sub-arrays were measured individually while already mounted within the panel. Mutual coupling is covered by the resulting embedded sub-array patterns. Based on these embedded patterns, the radiation pattern of the panel was calculated and compared to a measurement of the panel. In case of TerraSAR-X the model was validated on panel level with 32 sub-arrays and on leaf level with four panels (= 128 sub-arrays).

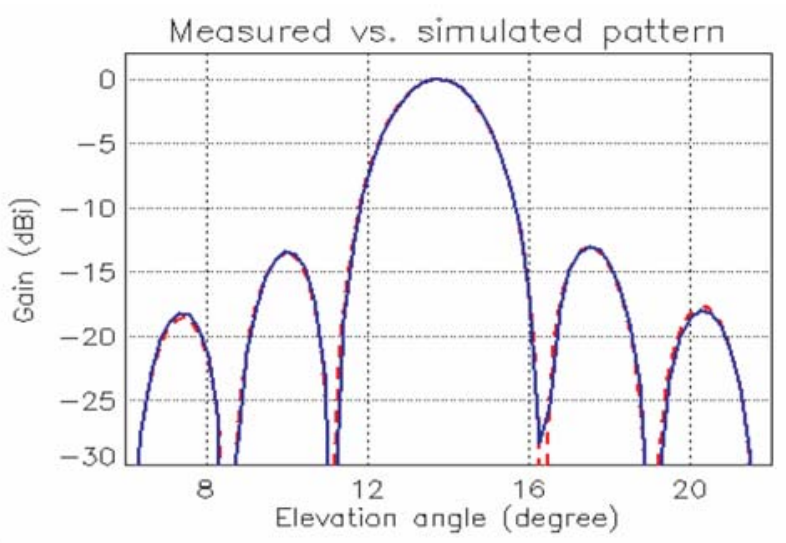

A)

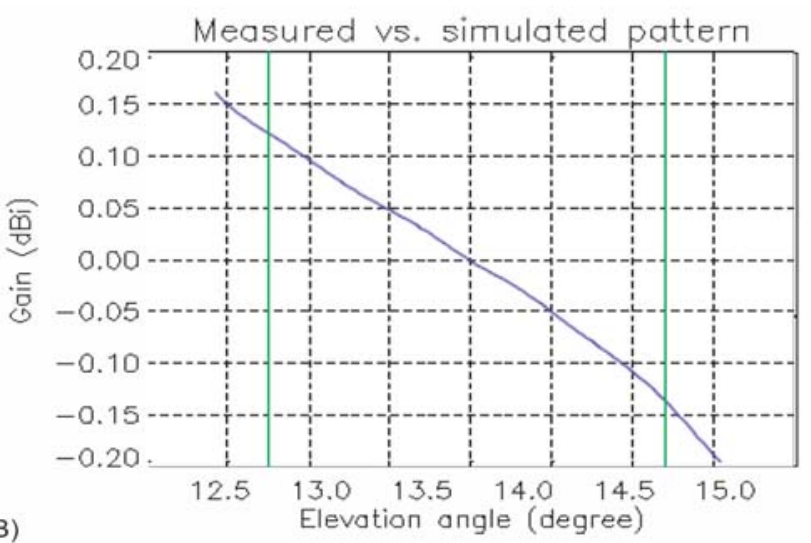

Figure 1: A) comparison between an exemplary beam measured on ground (blue/solid) and derived by the antenna model (red/dotted), B) difference within the main beam between model and measurement.

One example of the validation on-ground performed by Astrium is shown in Figure 1. The solid blue curve is the measured elevation pattern of one panel and the red curve of that derived by the model. The deviation of the shape within 
the main lobe is less than $0.15 \mathrm{~dB}$. The results of the validation of the antenna model are described in [6].

The in-orbit verification will be performed by three selected beams with low, medium and high incidence angles. For this, the elevation patterns are measured over rain forest and compared to the pre-calculated patterns. Furthermore, the transmitted pulses are recorded on-ground by deployed ground receivers. With this method the transmit pattern of the TerraSAR-X antenna can be measured in-flight verifying the one-way azimuth patterns. One example of really measured antenna patterns of the ASAR/ENVISAT instrument is shown in Figure 2. The reduced amplitudes indicate the switching of the instrument from beam to beam and the envelopes represent the one-way azimuth pattern of the corresponding beam.

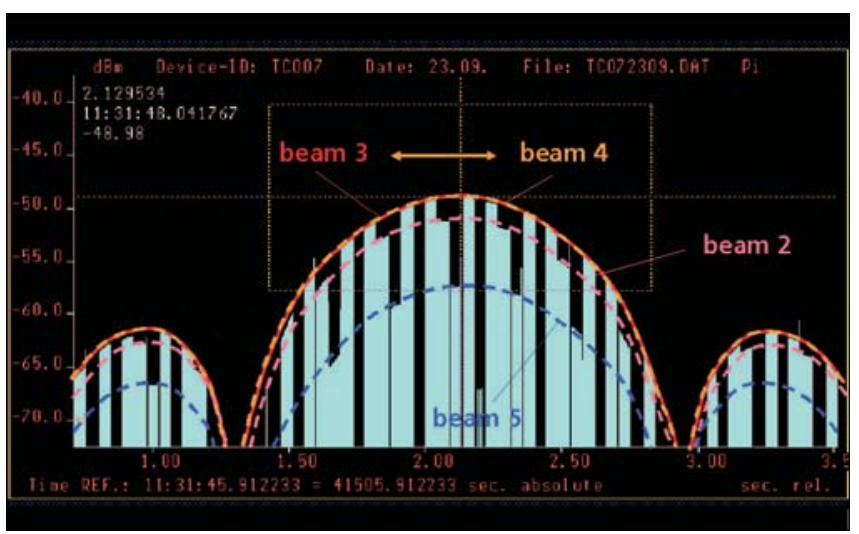

Figure 2: One-way (transmit) azimuth antenna patterns of the ASAR/ENVISAT instrument in ScanSAR operation measured by a ground receiver.

\subsection{Relative and Absolute Radiometric Calibration}

After in-orbit verification of the antenna model the beam patterns required for radiometric correction of SAR data within an illuminated scene are derived by the antenna model for all operation modes and all incidence angles. There are more than ten thousand possible antenna patterns und hence, the same amount of absolute calibration factors.

The relative gain variation from beam to beam will be characterised over homogeneous targets in ScanSAR operation. Thus, absolute radiometric calibration is sufficient for a few beams instead of measuring TerraSAR-X against deployed calibration targets for all operation modes. The effort for relative and absolute radiometric calibration as well as the duration of the calibration campaigns can be significantly reduced to achieve a five months commissioning phase.

\subsection{Antenna Performance Monitoring}

An important input to the model are the actual beam coefficients of the active phased array which can be monitored in-orbit using the novel T/R Module Characterisation Method [8]. Beyond these capabilities the antenna model also features a tool for generating optimised beam coefficients under given constraints. One major advantage of this beam optimisation is that the reference setting of the antenna excitation is already calculated before launch for the best instrument performance. After launch this optimisation method guarantees dynamic re-calibration in the event of active antenna module degradations during the mission. Figure 3 shows results from on-ground tests with the whole TerraSAR-X antenna for monitoring performance degradation of individual antenna modules.

Half an antenna panel (16 modules of panel number 10) was switched off whereas the whole antenna was excited with a gain taper in elevation (row) direction. The commanded receive gain taper in elevation applied to all panels is drawn as a solid line in Figure 3.

The T/R Module Characterisation Method is used to characterise the individual antenna beam coefficients while the instrument is operated under nominal conditions. The actual coefficients of antenna panels are compared to the commanded ones. The characterised gain of panel number 0 is plotted as a dashed line. It matches the commanded beam taper very well. Accordingly, the gain characteristics (dotted line) of antenna panel number 10 correspond to the expected values, too. The first 16 modules of antenna panel number 10 yield only noise signal as these modules were switched off. With the help of this method, a fast and dynamic recalculation of antenna beams is possible by feeding these inputs into the antenna model instead of measuring all antenna patterns again.

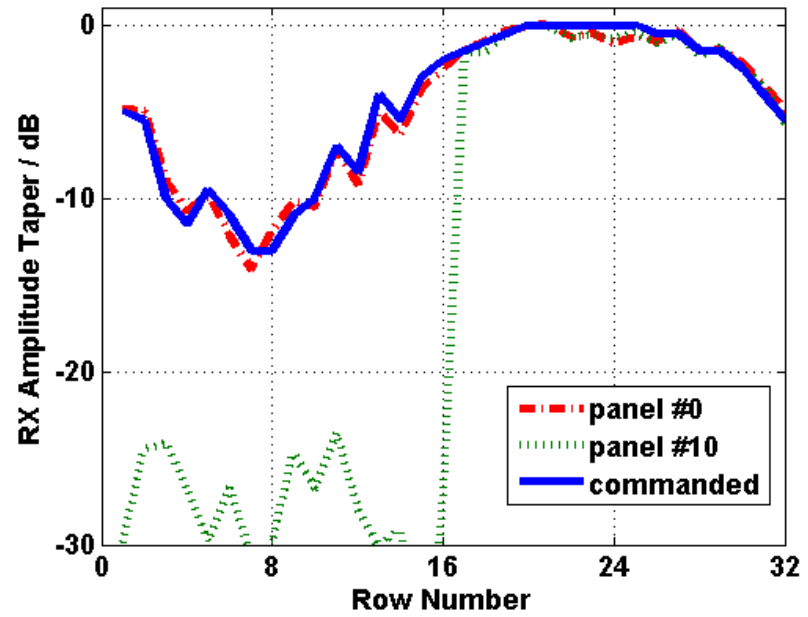

Figure 3: Commanded and characterised beam taper coefficients on antenna panel number 0 and number 10 of TerraSAR-X active antenna. The first 16 modules of panel 10 were switched off.

\section{Calibration Campaigns}

To ensure a successful execution of the different calibration procedures described above, dedicated calibration campaigns must be performed. This so called external calibration [10] is based on SAR data acquisition over test sites with wellknown backscatter characteristics. Basically, these sites can be homogenous areas like the Amazonian rain forest or sites with deployed point targets, like corner reflectors, active transponders or ground receivers. The most important driver to plan and coordinate these activities is the global coverage of TerraSAR-X, as the coverage defines the number of feasible measurements and consequently drives the schedule. 


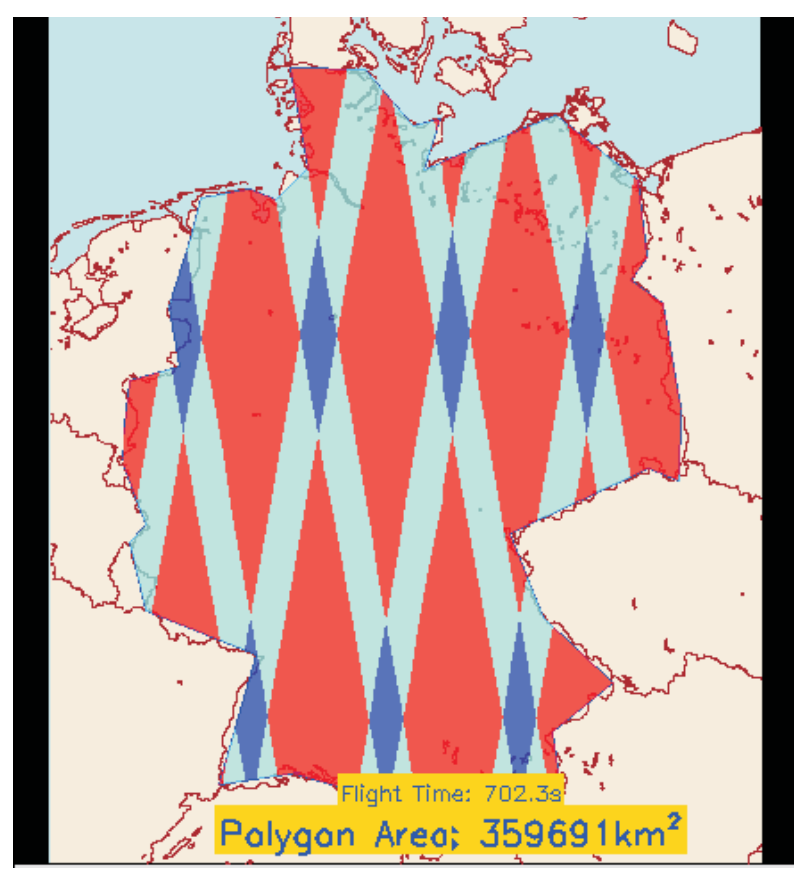

Figure 4: Coverage of TerraSAR-X StripMap beam strip011R over a complete repeat cycle. Red areas: no coverage, light blue areas: covered once, dark blue areas: covered twice.

\subsection{Global Coverage of TerraSAR-X}

The coverage on the earth's surface needs to be evaluated with respect to the required number of point target and rain forest measurements assumed in the radiometric accuracy budget. As single beams do not cover the complete globe, the selection of test sites is constrained to the availability of respective beams on ground. Figure 4 shows the coverage of the full performance beam 011 over Germany in right looking mode for a repeat cycle of eleven days. In order to obtain as many overflights as possible over calibration targets, special test site configurations have been selected in the crossing points of ascending and descending passes.

\subsection{Test Site Configuration}

Driven by the radiometric accuracy budget within a StripMap swath of $30 \mathrm{~km}$ range, two targets each are deployed in near range, at swath centre, and in far range, see Figure 5.

The targets must be sufficiently separated to avoid ambiguities in the image. With a slight displacement, most of the positions (red crosses) can be applied twice for descending and ascending orbit. Thus, the number of required target positions within a test site could be extremely reduced.

\subsection{DLR Calibration Field}

Mainly for logistic reasons test sites are selected near by DLR Oberpfaffenhofen in South Germany as calibration targets have to be re-adjusted for each overflight. According to the previously described test site configuration a total of 30 target positions have been established in an area of $120 \mathrm{~km}$ x $40 \mathrm{~km}$ covering several crossing orbits of TerraSAR-X antenna beams.

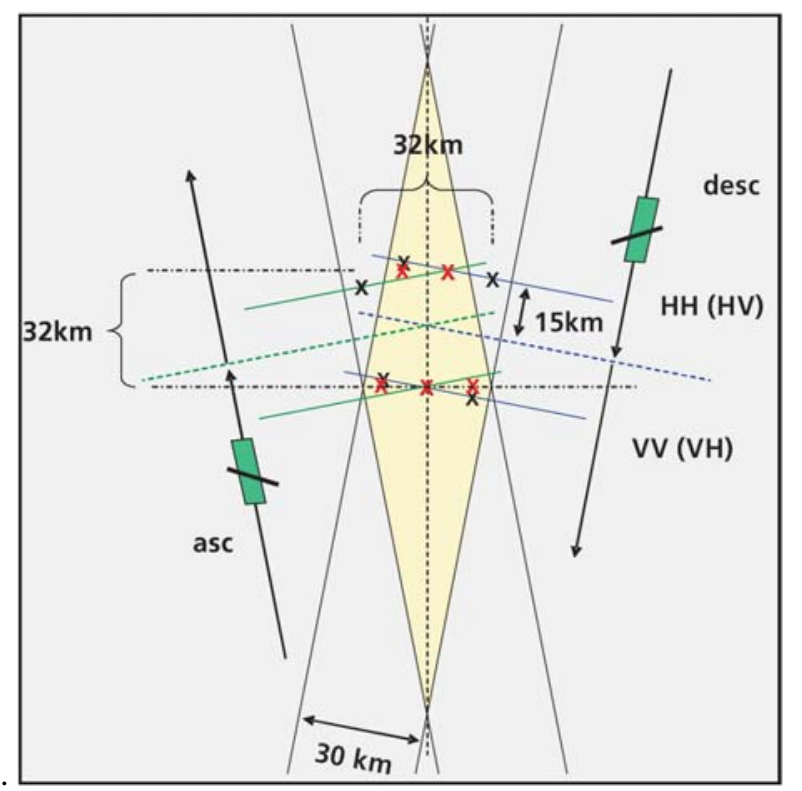

Figure 5: Test site configuration for TerraSAR-X. The test site consists of 7 target positions and encloses an area of $32 \mathrm{~km}$ x $32 \mathrm{~km}$.

Implementation of the calibration concept requires a calibration facility that is well-equipped with ground calibration hardware as well as software tools for evaluating the measurements. For external calibration, three different types of calibration targets are used:

- Trihedral and dihedral corner reflectors as passive targets precisely surveyed (with differential GPS) and therefore well suited for geometric calibration.

- Transponders with high radar cross section providing accurately defined point targets within the scene.

- Ground receivers measuring the one-way azimuth pattern of the SAR antenna during an overflight.

Different evaluation and analysis software tools have been tested during pre-launch preparation. The antenna model provides the validated antenna patterns and the on-board beam coefficients to be commanded in the SAR antenna. The T/R Module characterisation software demonstrated its valuable support of antenna performance monitoring already during on-ground instrument tests. For point target analysis the CALIX software features measurements of impulse response function parameters, absolute calibration factors as well as geometric analysis.

The existing infrastructure is well prepared for executing calibration campaigns over large test sites. Accordingly, reliable and accurate ground equipment as well as evaluation software is guaranteed during the whole mission life time. It should be mentioned that additional test sites are deployed in Northern Germany, Switzerland, and Spain.

\section{Summary}

In preparation of the TerraSAR-X satellite launch in June 2007 DLR has thoroughly planned the calibration procedures to be performed during the five months commissioning phase. 
By applying the antenna model as key element of the in-orbit calibration strategy, most of the antenna characterisation effort has been shifted from in-flight to on-ground activities. An efficient but accurate way to accomplish a short commissioning phase has been developed as only a few beams are measured in orbit.

A multitude of powerful software tools has been established within on-ground tests to support the evaluation of calibration measurements. For the first time ever, the innovative T/R Module characterisation method will be applied in a satellite environment during the TerraSAR-X mission. Evaluation of data acquired during the calibration campaigns yields different calibration and processing parameters like antenna patterns or absolute calibration factors. Therefore, corresponding test site configurations have been developed and arranged in South Germany, supported by precise and sophisticated ground equipment from the DLR Oberpfaffenhofen calibration facility.

After satellite launch calibration activities consume most of the time during the five months commissioning phase. The first measurements begin three weeks after launch. Over 400 acquisitions across the Amazonian rain forest are planned. The various calibration measurements in Germany require ground support by professional calibration teams performing more than 60 campaigns in a time frame of less than five months. This amount of measurements is sufficient for calibrating over 10,000 antenna beams.

The results from the in-orbit calibration are a pre-requisite for successful SAR product verification as they define the geometric and radiometric performance of TerraSAR-X.

\section{References}

[1] R. Werninghaus, "The TerraSAR-X Mission," in 6th European Conference on Synthetic Aperture Radar, Dresden, Germany, 2006.

[2] M. Schwerdt, B. Bräutigam, M. Bachmann, T. Molkenthin, D. Hounam, and M. Zink, "The Calibration of TerraSAR-X," in 6th European Conference on Synthetic Aperture Radar, Dresden, Germany, 2006.

[3] M. Schwerdt and D. Hounam, "Quality Control and Calibration of Future SAR Systems," in ODAS 2001, 3rd ONERA-DLR Aerospace Symposium, Paris, France, 2001.
[4] M. Schwerdt, D. Hounam, B. Bräutigam, and J. L. Alvarez-Pérez, "TerraSAR-X: Calibration Concept of a Multiple Mode High Resolution SAR,” in IEEE: 25th International Geoscience And Remote Sensing Symposium, Seoul, Korea, 2005.

[5] M. Zink and B. Rosich, “Antenna Elevation Pattern Estimation from Rain Forest Acquisitions," in ENVISAT/ASAR Calibration Review (ECR) of ESTEC, European Space Agency (ESA), Noordwijk, Netherland, 2002.

[6] M. Bachmann, M. Schwerdt, B. Bräutigam, B. Grafmüller, A. Herschlein, and J. L. Alvarez-Pérez, "The TerraSAR-X Antenna Model Approach," in 2nd International ITG Conference on Antennas, Munich, Germany, 2007.

[7] D. Hounam, M. Schwerdt, and M. Zink, "Active Antenna Module Characterisation by Pseudo-Noise Gating," in 25th ESA Antenna Workshop on Satellite Antenna Technology, Noordwijk, Netherlands, 2002.

[8] B. Bräutigam, M. Schwerdt, M. Bachmann, and M. Stangl, "Individual T/R Module Characterisation of the TerraSAR-X Active Phased Array Antenna by Calibration Pulse Sequences with Orthogonal Codes," in 26th International Geoscience And Remote Sensing Symposium, Barcelona, Spain, 2007.

[9] J. L. Alvarez-Pérez, M. Schwerdt, D. Hounam, R. Torres, C. Buck, and M. Zink, “Antenna Pattern Optimization for the ENVISAT ASAR Antenna with Failed Elements," in 25th ESA Antenna Workshop on Satellite Antenna Technology, Noordwijk, Netherlands, 2002.

[10] B. Bräutigam, M. Schwerdt, and M. Bachmann, "The External Calibration of TerraSAR-X, a Multiple Mode SAR-System," in 6th European Conference on Synthetic Aperture Radar, Dresden, Germany, 2006. 